\title{
Mutual-friction induced instability of normal-fluid vortex tubes in superfluid helium-4
}

\author{
Demosthenes Kivotides \\ University of Strathclyde Glasgow
}

(Dated: March 29, 2018)

\begin{abstract}
It is shown that, as a result of its interactions with superfluid vorticity, a normal-fluid vortex tube in helium-4 becomes unstable and disintegrates. The superfluid vorticity acquires only a small (few percents of normal-fluid tube strength) polarization, whilst expanding in a front-like manner in the intervortex space of the normal-fluid, forming a dense, unstructured tangle in the process. The accompanied energy spectra scalings offer a structural explanation of analogous scalings in fully developed finite-temperature superfluid turbulence. A macroscopic mutual-friction model incorporating these findings is proposed.
\end{abstract}




\section{PROLOGUE}

At temperatures smaller than $2.17 \mathrm{~K}$ (the lambda point), the quantum field that describes helium-4 becomes Bose-Einstein condensed, giving rise to a non-zero ground state that corresponds to an inviscid fluid ( "superfluid"). The superfluid coexists with the (classical-like) "normal-fluid" of the Bogoliubov quasiparticles that comprise the thermalized quantum fluctuations. Turbulence in such systems, "finite-temperature superfluid turbulence" or FTST for short, has a unique characteristic [1,2]: it is the only known type of turbulence, such that two different types of fluid-vortices interact with each other. These are the topological defects in the superfluid (linear vortices of quantized circulation), and the classical vortices in the normal-fluid. In FTST, superfluid and normal-fluid vortices interact via "mutual friction" forces $[3,4]$. Like any other problem in statistical physics, FTST can be studied in either of the Liouville/Hamiltonian representations, that are analogs of the Schroedinger/Heisenberg representations of quantum mechanics, and in dissipative, normal-fluid turbulence context consist of the familiar Hopf/Navier-Stokes formulations. Notably, the "realization" $(\mathcal{R})$ formulations (Hamilton/Heisenberg/Navier-Stokes) can only be interpreted stochastically, i.e., subject to random initial conditions, they provide the means to generate sample paths of the random fields (e.g., velocity and pressure in turbulence) whose ensemble averages are the key objectives of the theory, and the only quantities of empirical value. In turbulence research, an ergodic hypothesis allows the inference of ensemble averages via spatial averaging. On the other hand, the "probabilistic" $(\mathcal{P})$ formulations (Liouville/Schroedinger/Hopf) are closed, but in the context of analytically intractable problems like turbulence, they face difficulties in providing closed statistical moment equations that can accurately capture the effects of vortical coherent structures and their interactions (the "turbulence problem"). For this reason, the present research employs the $\mathcal{R}$ formulation of FTST, and, by directly calculating the interactions between vortical structures in both fluids, draws conclusions about its statistical structure. This approach has a long tradition in classical turbulence theory [5-7]. For example, [8] indicated that a system of viscous, reconnecting vortex tubes reproduces the Kolmogorov $k^{-5 / 3}$ scaling of inertial range turbulence, and [9] showed that systems of vortex elements give rise to Levy rather than Gaussian distributions for turbulent flow velocity. Similar connections between vortices and spectra [10-12], as well as, vortices and velocity distributions $[13,14]$ were also obtained in quantum fluids. In FTST, [12] showed 

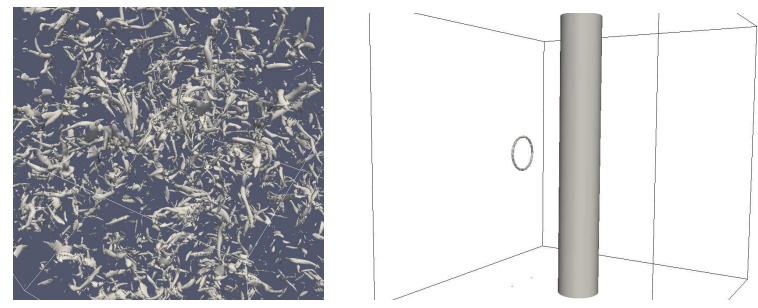

FIG. 1. Left: Vorticity magnitude isosurfaces at level equal to 0.35 times its maximum value, for a homogeneous, isotropic, pure normal-fluid turbulence at Taylor Reynolds number $R e_{\lambda} \approx 100$. Although sheet-like structures are present, we predominantly see linear vortices. Right: The initial configuration consists of a straight normal-fluid vortex tube of circulation strength $\Gamma=20 \times 10^{3} \nu$, and a superfluid ring of circulation strength $\kappa \approx \Gamma / 46,747$.

that interactions between one normal-fluid and many superfluid vortex rings generate a tendency towards energy-level matching in the wavenumber intervals of the velocity spectra which correspond to the normal-fluid ring diameter scale. Remarkably, there is no accompanying vorticity matching, because, for typical normal-fluid Reynolds numbers, the inertia of the normal-fluid ring is much stronger than mutual-friction effects on superfluid vortices, hence, the latter cannot be coaxed into aligning with the former within the time-scales of normal-fluid ring motion. Fig.1 (left) shows the isosurfaces of enstrophy in homogeneous, isotropic Navier-Stokes turbulence of Taylor Reynolds number $R e_{\lambda} \approx 100$. The results have been produced with a projection-type, incompressible Navier-Stokes solver with periodic boundary conditions. The results of Fig.1 indicate that the normal-fluid vorticity within the inertial range of turbulence has a predominantly linear (rather than ring-like) structure, and although the vortices are curved and expected to move, the normal-fluid vortex motion effect is not as vigorous as in [12]. Perhaps then, a straight tube could be a helpful (albeit approximate) model for the normal-fluid vortex structures shown in Fig.1, in the sense, that, since a straight tube does not move, superfluid vorticity polarization effects would be present in the highest degree possible. In other words, one would reasonably anticipate the phenomenology of actual FTST to lie in between the ring [12] and straight tube cases.

Notably, straight tube/superfluid vorticity interactions have been studied before [10, 15], yet these studies are incomplete from the physics point of view, since they employ a prescribed normal-flow, and ignore the effects of mutual-friction on the latter. In this work, 
we account for the full physics, since we employ the mesoscopic model of superfluid dynamics [3], that describes the interactions between (turbulent) vortex structures and individual topological defects. We shall indicate that the combination of present and previous findings of the mesoscopic model lead to a novel formulation of the macroscopic (i.e., assuming a continuous superfluid vorticity field) equations of superfluid dynamics. In particular [16, 17], there are presently two macroscopic prescriptions for the mutual-friction force per unit volume $\mathbf{f}_{\mathrm{MF}}$ (as it appears in the equation for the normal-fluid): (a) the Gorter and Mellink $(\mathrm{GM})$ formula $\mathbf{f}_{\mathrm{GM}}=-\rho_{s} \rho_{n} A V_{n s}^{2} \mathbf{V}_{n s}$, where $\rho_{s}$ and $\rho_{n}$ are (correspondingly) the superfluid and normal-fluid mass densities, $\mathbf{V}_{n s}=\mathbf{V}_{n}-\mathbf{V}_{s}$, where $\mathbf{V}_{n}, \mathbf{V}_{s}$ are (correspondingly) the normal-fluid and superfluid velocities, $V_{n s}$ is the magnitude of $\mathbf{V}_{n s}$, and $A$ is a function of the temperature $T$ and $V_{n s}$. The GM formula is consistent with a chaotic, isotropic, superfluid vortex tangle, hence, a tangle whose organization does not mimic the (inertial range) vortex structure of normal-fluid turbulence shown in Fig.1, (b) the Hall-Vinen-BekharevichKhalatnikov $(\mathrm{HVBK})$ formula $\mathbf{f}_{\mathrm{HVBK}}=\frac{B \rho_{s} \rho_{n}}{\rho \omega_{s}} \boldsymbol{\omega}_{s} \times\left(\boldsymbol{\omega}_{s} \times \mathbf{V}_{n s}\right)+\frac{B^{\prime} \rho_{s} \rho_{n}}{\rho} \boldsymbol{\omega}_{s} \times \mathbf{V}_{n s}$, where $B$, $B^{\prime}$ are macroscopic mutual-friction parameters that depend on temperature, second sound frequency, and flow velocity, $\rho=\rho_{s}+\rho_{n}, \boldsymbol{\omega}_{s}$ is the continuous superfluid vorticity field, and $\omega_{s}$ its magnitude. Although the HVBK formula has also been employed in homogeneous, isotropic turbulence situations, it is, in principle, a model of rotating superfluid turbulence, since it assumes a highly organized superfluid vorticity state, in the form of superfluid vortex bundles that mimic normal-fluid vorticity structures. Although the organization of flow vorticity into columnar structures of large-eddies that are parallel to the rotation axis is typical of rotating turbulent flows [5], we shall see here that, in the absence of rotation, they do not follow from more microscopic formulations of superfluid dynamics. The aforementioned presuppositions carry over to the vortex dynamics of the HVBK equations as formulated and solved in the pioneering contributions of Schwarz [18]. It is important to note here that, since HVBK vortex dynamics refers to the vortex lines in the continuous superfluid vorticity field, its comparison with experiments that measure the vortex line density of discrete topological defects tangles (that, moreover, do not obey organization assumptions embodied in the HVBK equations), is not methodologically sound. The usefuleness of HVBK vortex dynamics (as a means for understanding the structure of superfluid turbulent flows) becomes even more questionable when we notice that, in previously published papers following this approach, the normal-fluid is kinematically prescribed, instead of been dynamically resolved 
via the HVBK equation for the normal-fluid. The latter shortcoming (which is on top of the aforementioned HVBK presuppositions) is a very important one, since, as we shall demonstrate here, the effects of superfluid back-reaction on normal-fluid vortices are simply too important to be ignored $[3,4,12]$. The mesoscopic model on the other hand, describes individual topological defects whose dynamics are coupled with the Navier-Stokes equations and not with the HVBK equation for the normal-fluid. Because of these characteristics, the mesoscopic model has predicted quantities in direct correspondence and impressive agreement with experiments. These are the prediction of tracer particle velocities in thermal counterflow turbulence [19, 20], and the temporal scaling for the superfluid vortex line density in grid turbulence decay [4] experiments. As we shall see, a combination of current and previous mesoscopic model results indicate that neither GM or HVBK formulas are directly applicable to homogeneous, isotropic superfluid turbulence. Instead, we propose here a new formula for macroscopic mutual-friction effects that takes into account topological defect curvature and superfluid vorticity intensity factors. An important goal is to explain the phenomenology of, recently performed, fully resolved superfluid turbulence calculations $[3,4]$ by analysing in great detail the physics of key elementary vortex processes in superfluid turbulence.

\section{MATHEMATICAL MODEL AND SOLUTION METHODS}

As mentioned above, the $\mathcal{R}$ formulation of superfluid turbulence follows here the incompressible, mesoscopic model of refs. [3, 4]. In this formulation, discrete (albeit coarsegrained) topological defects (vortices) in the condensate, interact with a normal-fluid continuum. The motion of point $\mathbf{X}_{v}(t)$ belonging to the superfluid vortex tangle $\mathcal{L}$ is governed by the zero sum of (from start to end) Magnus, Hall-Vinen, Iordanskii, and reconnection forces

$$
\begin{array}{r}
\rho_{s} \kappa \mathbf{X}_{v}^{\prime} \times\left(\mathbf{V}_{s}-\dot{\mathbf{X}}_{v}\right)+D_{0} \mathbf{X}_{v}^{\prime} \times\left[\mathbf{X}_{v}^{\prime} \times\left(\mathbf{V}_{n}-\dot{\mathbf{X}}_{v}\right)\right]+ \\
\rho_{n} \kappa \mathbf{X}_{v}^{\prime} \times\left(\mathbf{V}_{n}-\dot{\mathbf{X}}_{v}\right)-\int_{\mathcal{L}} d\left|\mathbf{X}_{\mathcal{L}}\right| \mu_{v} \ddot{\mathbf{R}} \delta\left(\left|\mathbf{X}_{v}-\mathbf{X}_{\mathcal{L}}\right|\right)=0 .
\end{array}
$$

Here, $\mu_{v}$ is the vortex mass per unit length, $\mathbf{X}_{v}^{\prime}$ the unit tangent to the line vortices, $\rho_{s}$ the superfluid mass density, $\kappa$ the quantum of circulation, $\rho_{n}$ the normal-fluid mass density, $D_{0}$ the coefficient of the Hall-Vinen force, $\mathbf{V}_{s}$ the Biot-Savart velocity, $\mathbf{V}_{s}\left(\mathbf{X}_{v}\right)=\frac{\kappa}{4 \pi} \int_{\mathcal{L}} \frac{\left(\mathbf{x}-\mathbf{X}_{v}\right) \times d \mathbf{x}}{\left|\mathbf{x}-\mathbf{X}_{v}\right|^{3}}$, 
and $\mathbf{R}$ a deterministic, pointwise-exact, reconnection-jump process that models the topological (i.e., cut and glue) transition from one smooth superfluid tangle configuration to another $[3,21]$. It is important to note here that, although this equation does not include vortex inertia/acceleration, it includes the vortex mass per unit length $\mu_{v}$ in the formal term depicting reconnections. This is not an inconsistency, since in the reconnection term, $\mu_{v}$ multiplies $\ddot{\mathbf{R}}$ which is an (instantaneous) jump process, that is not resolved at the mesoscopic range of scales of interest here, hence, it does not contribute to the vortex acceleration. In other words, since the reconnection process is written formally as a jump process, it is not dynamically resolved (and the actual $\mu_{v}$ value is not relevant). Instead, the computational algorithms perform the topological changes at the level of the data-structures that enunciate the connectivity of the superfluid vortices, within every-time step, and after the computation of mutual-friction effects in the vortex dynamical equation above. Explicit details of these procedures are discussed in ref. [21].

The normal-fluid obeys standard Navier-Stokes dynamics, i.e., the mass equation $\nabla \cdot \mathbf{V}_{n}=0$, and the momentum equation

$$
\begin{aligned}
& \frac{\partial \mathbf{V}_{n}(\mathbf{x}, t)}{\partial t}+\nabla\left(\frac{p}{\rho_{n}+\rho_{s}}+\frac{\mathbf{V}_{n} \cdot \mathbf{V}_{n}}{2}\right)-\mathbf{V}_{n} \times\left(\nabla \times \mathbf{V}_{n}\right)- \\
& \frac{\mu}{\rho_{n}} \nabla^{2} \mathbf{V}_{n}-\kappa \int_{\mathcal{L}} d\left|\mathbf{X}_{\mathcal{L}}\right|\left[\mathbf{X}_{\mathcal{L}}^{\prime} \times\left(\mathbf{V}_{n}-\dot{\mathbf{X}}_{\mathcal{L}}\right)\right] \delta^{3}\left(\mathbf{x}-\mathbf{X}_{\mathcal{L}}\right)- \\
& \frac{D_{0}}{\rho_{n}} \int_{\mathcal{L}} d\left|\mathbf{X}_{\mathcal{L}}\right|\left\{\mathbf{X}_{\mathcal{L}}^{\prime} \times\left[\mathbf{X}_{\mathcal{L}}^{\prime} \times\left(\mathbf{V}_{n}-\dot{\mathbf{X}}_{\mathcal{L}}\right)\right]\right\} \delta^{3}\left(\mathbf{x}-\mathbf{X}_{\mathcal{L}}\right)=0 .
\end{aligned}
$$

Here, $p$ is the pressure field, and $\mu$ the normal-fluid viscosity (with $\mu / \rho_{n}=\nu$ the kinematic viscosity). From start to end, we have the local acceleration, potential ("Bernoulli-group"), vortex, dissipative, Iordanskii and Hall-Vinen forces. In this work, we produce approximate solutions of these equations employing the numerics and algorithmics discussed in [3].

\section{INITIAL AND BOUNDARY CONDITIONS AND CALCULATION SPECIFICS}

The initial configuration (Fig.1) consists of a straight normal-fluid vortex tube of circulation strength $\Gamma=20 \times 10^{3} \nu$, and a superfluid ring of circulation strength $\kappa \approx \Gamma / 46,747$ placed in a periodic box of size $l_{b}=0.1 \mathrm{~cm}$. The chosen $\Gamma$ value is typical of inertial range coherent vortices in a turbulent normal-fluid. The vorticity distribution within the tube is Gaussian [22], with standard deviation $\sigma=l_{b} / 16$ (the "tube radius"). The superfluid ring 
(whose radius is also equal to $R=l_{b} / 16$ ) is initially positioned on a plane parallel to the tube axis, at distance $l_{b} / 4$ from the box-centre. It is important to note here, that the final conclusions are independent of the particular initial conditions. This is because, as discussed below, the conclusions emanate from generic physical principles, and are based on turbulence states that result from an enormous amplification of seed superfluid vorticity, which is accompanied by hundreds of thousands of reconnections, that result in a complete loss of correlations with the initial conditions. The Navier-Stokes grid size is chosen to be same as the discretization length along superfluid vortices, and equal to $\Delta x=l_{b} / 128$. Two vortices reconnect when they approach each other closer than distance $0.75 \min (\Delta x, \lambda)$, where $\lambda$ is the average intervortex distance in the tangle.

In order to characterize better the normal-fluid flow regime, we introduce three nondimensional parameters: (a) the normal fluid Reynolds number $R e=\Gamma / \nu$, that measures the ratio of inertial and viscous forces, and, since $R e=20 \times 10^{3}$, it indicates that (as is typical of turbulence) inertia dominates viscous effects in the vortex-tube flow, (b) the interaction parameter $N_{1}$, that is analog of a related concept in plasma flows [23], and measures the ratio of inertial and Iordanskii forces, $N_{1}=\left(u^{2} / \ell\right) /\left(\kappa u / \xi^{2}\right)=u \xi^{2} / \kappa \ell$, where $\xi$ is the effective length scale below which the topological effects are smoothed out, loosing their linelike character, and is taken to be equal to $\Delta x$, the computational grid size. A detailed discussion of $\xi$ is available in ref.[3]. Moreover, $\ell$ is a length scale that characterizes the large scale flow motions (for example, it can be chosen to be half of the box size), and $u$ is the characteristic velocity scale at the length scale $\ell$, that can be computed via the relation $u \ell=\Gamma$. Inserting the appropriate numbers in, we find $u=932.12 \mathrm{~cm} / \mathrm{s}$ and $N_{1}=11.378$. In a similar fashion, we can define (c) a second interaction parameter $N_{2}$ corresponding to the Hall-Vinen drag force, $N_{2}=u \xi^{2} / \zeta \ell$ (where $\zeta=D_{0} / \rho_{n}$ ), which is equal to $N_{2}=2.285$. Notably, in deriving the interaction parameters, we used the fact that the initial superfluid vortex line density is very small, so that $\mathbf{V}_{n}-\dot{\mathbf{X}}_{\mathcal{L}}$ can be replaced with $\mathbf{V}_{n}$. The two interaction parameters are very informative: they explain why, despite the fact that the initial superfluid vortex line density is very small, locally, mutual friction excitation appears significant when compared to flow inertia. Hence, one could reasonably expect non-negligible effects of Iordanskii and Hall-Vinen forces on the normal-fluid turbulence structures. 

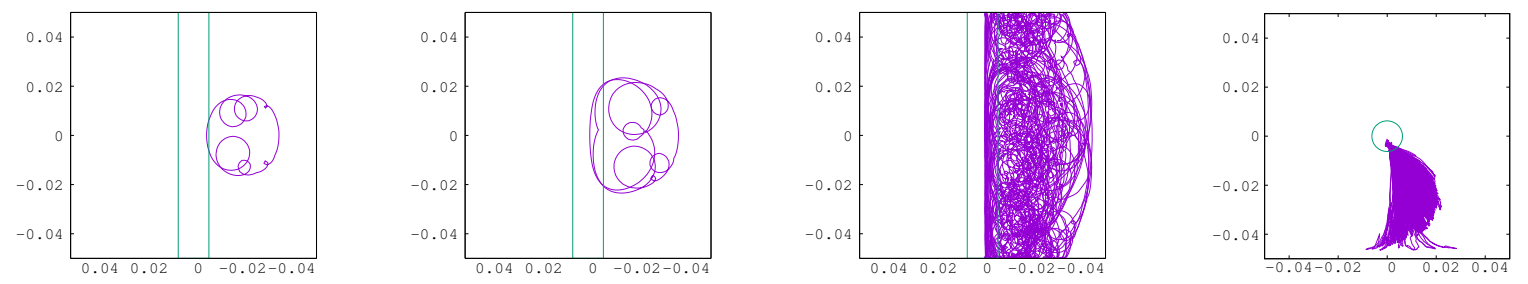

FIG. 2. Left to Right: Superfluid vorticity tangle at times $t=[2.78,3.48,9.81] \times 10^{-4} \mathrm{~s}$ (side view) and $t=12.4 \times 10^{-4} \mathrm{~s}$ (top view). The two vertical lines in the first three figures from the left delineate the boundaries of the initial vortex tube, as does the circle in the top view figure. Notably, the region occupied by the tangle terminates along the initial tube axis, and the tangle's expansion along the perimeter occurs mostly outside the vortex tube, due to the differential rotation effect there.
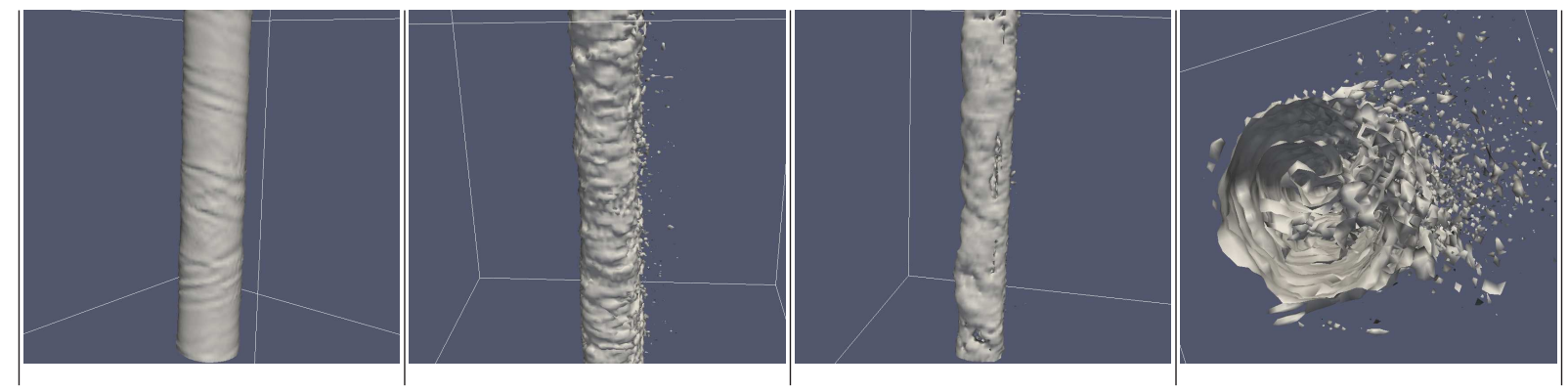

FIG. 3. Left to Right: Normal-fluid vorticity magnitude isosurfaces at times $t=[7.3,12.4,15.8] \times$ $10^{-4} \mathrm{~s}$ (side view) and $t=15.8 \times 10^{-4} \mathrm{~s}$ (top view). The corresponding (left to right) vorticitymagnitude levels are $[60,70,70,105] \times 10^{3} \mathrm{~s}^{-1}$.

\section{COMPUTATIONAL SOLUTION}

In order to understand the results, it is important to discern two regions in the normalflow: (a) the region within the tube radius, where the normal-fluid rotates like a solid body, and (b) the region outside, where the flow is potential, and, as a result, fluid layers rub against each other. As shown in Fig.2, the tube flow field first re-orients the ring, so that part of it now sits on a plane normal to the azimuthal direction, hence, taking into account the flow streamlines, the Hall-Vinen force blows up the ring towards the tube axis, tending to align (this way) normal-fluid and superfluid vorticities. In the process, Ostermeier-Glaberson vortex instabilities $[10,15]$ generate small wiggles that grow to large sizes themselves. As the loops multiply, blown up cycles meet each other and reconnect, producing unstruc- 

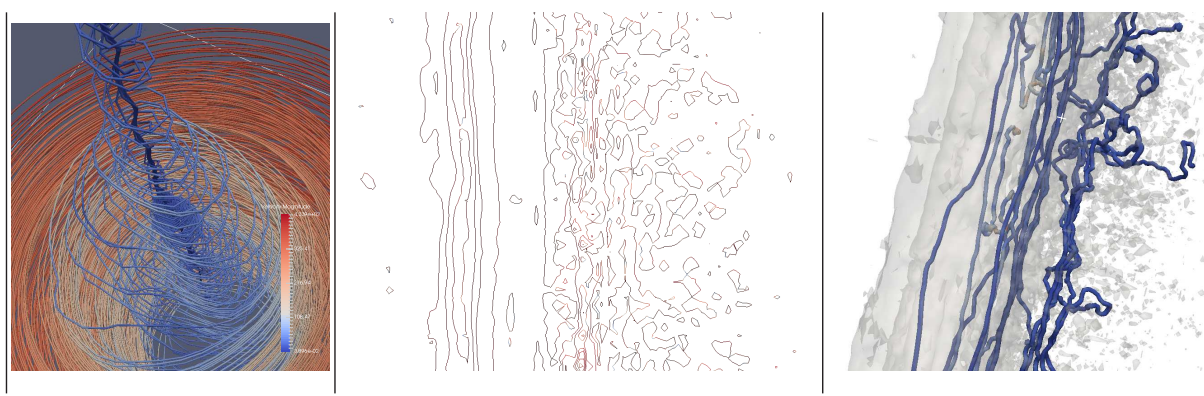

FIG. 4. Left to Right: (a) Normal-fluid streamlines within the tube core at $t=13.83 \times 10^{-4} \mathrm{~s}$, (b) normal-fluid vorticity-magnitude isolines on a plane that includes the tube axis and passes through the superfluid tangle domain; fourteen levels between $24 \mathrm{~s}^{-1}$ and $588385 \mathrm{~s}^{-1}$ values, at $t=16.02 \times 10^{-4} \mathrm{~s}$, are shown; in opposition to the straight lines in the initial state, we observe closed isolines within the tube, as well as fine-grained patterns in the superfluid tangle domain. (c) normal-fluid vortex lines passing between the tube centre and its edge, and superimposed onto the background of an enstrophy isosurface; some extend along the tube axis (as in the initial state), but others change direction, form spirals, and/or wander into the superfluid tangle domain (fine-structure enstrophy region in the background).
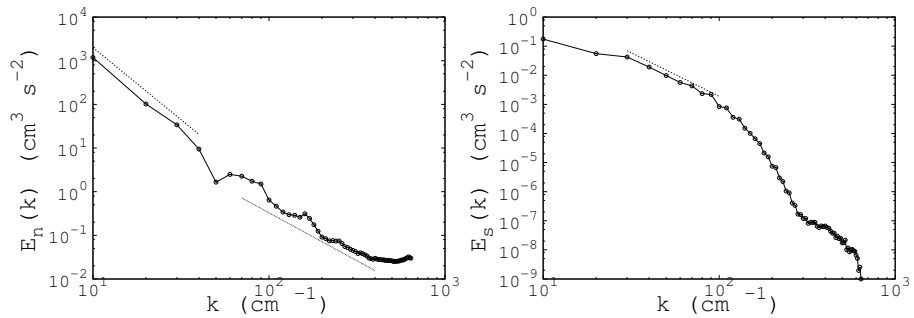

FIG. 5. Velocity spectra at $t=16.02 \times 10^{-4} \mathrm{~s}$, for normal-fluid (left) and superfluid (right). The two normal-fluid scalings shown are $k^{-3.3}$ (low $k$ ), and $k^{-2.2}$ (high $k$ ), whilst the mesoscopic superfluid scaling is $k^{-3}$.

tured, small scale vorticity. As shown in the final Fig.2 picture, since the normal-fluid near the tube axis (but still within the potential flow region) rotates faster than the fluid far away, and under the influence of the Iordanskii force, the superfluid vorticity cloud rolls up spirally, acquiring also (due to self-induction or initial set-up memory effects) an ever increasing thickness along the azimuthal. In the end of the calculation $\left(t=16.02 \times 10^{-4} \mathrm{~s}\right)$, 
the magnitude of superfluid vorticity within the tube is approximately $1 \%$ of the tube's strength. Notably (Fig.2), the superfluid vorticity alignment with the normal-fluid vorticity takes place exclusively within the tube radius. In conclusion, there are two superfluid vorticity regions: one within the solid body rotation core where mild polarization occurs, and another outside, where the bulk of vortex-tangle length is in an unstructured/chaotic state, and superfluid-vorticity propagation into the normal-fluid intervortex space of Fig.1 (left) resembles a traveling front. This mode of superfluid vorticity propagation was also obtained in the [21] calculations.

A most important finding, relates to the normal-fluid (Fig.3): the tube becomes unstable (first picture on the left), and a system of helical, normal-fluid vorticity ( $\boldsymbol{\omega}=\nabla \times \mathbf{V}_{n}$ ) waves appear. Such a wave system is modeled via the helical wave decomposition [24] of divergencefree vectors. A similar phenomenon was discovered in pure normal-fluid tube dynamics [25]. There are some similarities: in both cases, the core evolution can be analyzed in terms of propagation/interaction of right-handed $\boldsymbol{\omega}_{R}$ and left-handed helical waves $\boldsymbol{\omega}_{L}$, that dominate different parts of the tube, i.e., there is no mix of different polarity waves (except at the centre where they collide). However, [25] had to introduce a sinusoidal axial perturbation of the tube-core size in order to kick-start the instability, whilst, in the FTST case, this is not necessary, since the instability is induced via mutual-friction force excitation. Indeed, there is a concentration (Fig.2) of (straight) superfluid vortices within the solid-body rotation region, which, via the mechanism indicated in [26], generate there dissipative vortical structures in the normal-fluid. As a result, the tube-core flow is distorted, giving rise to (1) a nontrivial vorticity structure (Fig.3, right), (2) spiraling streamlines (Fig.4, left) that converge towards the tube axis, and (3) a near-stagnation flow blob (same picture), also located on the tube axis. The latter feature is not accidental, since it is well known from classical vortex dynamics [27], that "vortex breakdown" is characterized by the formation of an internal (within the core) stagnation point on the vortex axis. In FTST, superfluid vorticity polarization is the driving force behind the above mentioned effects, but there is a nonlinear feedback mechanism, since the onset of polarization helps destroy (via induced instability) the flow field responsible for it in the first place. This feedback mechanism offers a good explanation for the observed small strength of polarization effect. As instability develops (second graph in Fig.3), the undulations of tube vorticity isosurfaces become more and more pronounced, until (third graph in Fig.3) strong deviations of tube shape (including a few 
"scars") from the initial cylindrical geometry are observed. The last graph in Fig.3 shows a large asymmetry in the tube-core structure, accompanied by a prominent cloud of fine-grain vorticity. The latter is also due to the [26] vorticity generation mechanism, and directly corresponds to the superfluid-vorticity propagating front of Fig.2. Remarkably, despite the fact that the initial straight tube has no self-induced motion, there is a net displacement of the tube towards this fine-grain vorticity region. The complex vorticity structure within the core is evident in the vorticity isolines of picture Fig.4 (middle). The highest of the local maxima shown there correspond to either spiral vortex-line structures, or regions of high vortex-line twist/curvature (Fig.4, right). Notably, the latter picture depicts also vortexline direction reversals within the tube-core, whilst the background tangle corresponds to vortex-lines meandering into the fine grain vorticity region of Fig.3 (right). It is important to note that (at final time) the maximum vorticity magnitude is more than two times larger than the corresponding value in the initial data.

The normal-fluid velocity spectra (Fig.5, left) show a steep $k^{-3.3}$ scaling tendency at low wavenumbers, that corresponds to the large-scale tube flow field, since it is also observed in the initial data. The $k^{-2.2}$ high wavenumbers scaling corresponds to the cloud of small scale (normal-fluid) vorticity created by the superfluid vorticity front, and it is in full agreement with the scaling found in $[3,4]$, and attributed there to dissipative, small-scale, normal-fluid flow induced by a complex superfluid vortex tangle via mutual friction excitation. It is informative to note here, that the sharper-slope interval in-between the two scaling regimes mentioned above corresponds to the $k^{-5}$ scaling law indicated in [28]. The $k^{-5}$ scaling is much more clearly discernible in the initial data, since they are not "contaminated" by mutual-friction effects. The appearance of this scaling close to the tube diameter wavenumber $\left(k=80 \mathrm{~cm}^{-1}\right)$, is also consistent with [28]. The superfluid spectrum (Fig.5, right) presents a $k^{-3}$ scaling regime at mesoscopic wavenumbers. This scaling has also been found in $[3,4,21]$, and was attributed there to the growth process of small, instability induced superfluid vorticity loops, noting that it characterizes the superfluid tangle in between the normal-fluid vortices. This picture is fully supported here, since the superfluid loops in the range of scales where the $k^{-3}$ scaling appears belong to the propagating front, i.e., they live in between the vortices of Fig.1 (left). 


\section{EPILOGUE}

At this point, the mesoscopic model has made a number of key, experimentally confirmed, predictions [4, 19, 20, 29-31], and resolved the structure of grid superfluid turbulence [3, 12]. By solving its equations, it has been indicated here that previous assumptions in the macroscopic modeling of normal-fluid/superfluid vortex interactions are not supported by this, more microscopic, methodology. In summarizing the implications of the mesoscopic approach in the modeling of superfluid dynamics, we note that its predictions suggest that macroscopic modeling of mutual friction force $\mathbf{f}_{\mathrm{MF}}$ ought to involve a combination of the structured HVBK $\left(\mathbf{f}_{\mathrm{HVBK}}\right)$ and chaotic Gorter-Mellink $\left(\mathbf{f}_{\mathrm{GM}}\right)$ closures. In particular, defining suitable thresholds $\omega_{s}^{t}$ and $\omega_{n}^{t}$ for corresponding superfluid and normal-fluid vorticity magnitudes, and denoting by $K_{n}$ the curvature of normal-fluid vorticity lines, we have that

$$
\begin{aligned}
\mathbf{f}_{\mathrm{MF}}= & H\left[\omega_{s}-\omega_{s}^{t}\right] \mathbf{f}_{\mathrm{HVBK}}+\left(1-H\left[\omega_{s}-\omega_{s}^{t}\right]\right) \\
& \left\{H\left[\omega_{n}-\omega_{n}^{t}\right]\left[h\left(K_{n}\right) \mathbf{f}_{\mathrm{GM}}+\left(1-h\left(K_{n}\right)\right) \mathbf{f}_{\mathrm{HVBK}}\right]+\left(1-H\left[\omega_{n}-\omega_{n}^{t}\right]\right) \mathbf{f}_{\mathrm{GM}}\right\} .
\end{aligned}
$$

Here, $H[\omega]$ is the Heaviside function, which is equal to one for positive $\omega$ and zero otherwise, and $h(K)$ a function that satisfies $h(K) \rightarrow \beta$ when $K \ll 1$, and $h(K) \rightarrow 1$ when $K \gg 1$. According to the physics of this formula, flow points with high $\omega_{s}$ values correspond to a structured vortex tangle, and their mutual-friction needs to be handled via $\mathbf{f}_{\mathrm{HVBK}}$. In lower $\omega_{s}$ regions, the determining factor is $\omega_{n}$ : at flow points with small $\omega_{n}$ values, the tangle polarization is negligible, and $\mathbf{f}_{\mathrm{GM}}$ needs to be employed, whilst at points with high $\omega_{n}$ values, a mild tangle polarization is expected that depends on the curvature of normal-fluid vorticity-lines, as discussed here and in [12]. Indicative parameter values suggested by the mesoscopic model are $\beta \approx 0.95, \omega_{n}^{t} \approx 0.8 \omega_{n}^{\max }$, and $\omega_{s}^{t} \approx 0.05 \omega_{n}^{t}$.

From another point of view, the main result in the manuscript indicates that HVBK dynamics cannot be possibly correct in case of homogeneous, isotropic superfluid turbulence, since the assumed organization of superfluid vortices via mimicking of normal-fluid vortex structures is not viable: the actual process of superfluid vorticity imitation of coherent normal-fluid vorticity destroys the integrity of the latter. 


\section{ACKNOWLEDGEMENTS}

I am very grateful to Tony Leonard for valuable discussions, and Caltech's Aerospace Department (GALCIT) for their generous hospitality during my visiting faculty appointment.

[1] R.J. Donnelly, Quantized Vortices in Helium II, Cambridge University Press (2008).

[2] S.K. Nemirovskii, Physics Reports 524, 85-202 (2013).

[3] D. Kivotides, Phys. Fluids 26, 105105 (2014).

[4] D. Kivotides, J. Low Temp. Phys. 181, 68 (2015).

[5] P.A. Davidson, turbulence, OUP (2004).

[6] A.A.R. Townsend, The Structure of Turbulent Shear Flow, Cambridge University Press (1975).

[7] D.I. Pulin, P.G. Saffman, Annu. Rev. Fluid Mech. 30, 31 (1998).

[8] D. Kivotides, A. Leonard, Phys. Rev. Lett. 90, 234503 (2003).

[9] I.A. Min, I. Mezic, A. Leonard, Phys. Fluids 8, 1169 (1996).

[10] D. Kivotides, S.L. Wilkin, J. Low Temp. Phys. 156, 163 (2009).

[11] S.K. Nemirovskii, Phys. Rev. B 90, 104506 (2014).

[12] D. Kivotides, EPL 112, 36005 (2015).

[13] M.S. Paoletti et al., Phys. Rev. Lett. 101, 154501 (2008).

[14] A.C. White et al., Phys. Rev. Lett. 104, 075301 (2010).

[15] D.C. Samuels, Phys. Rev. B 47, 1107 (1993).

[16] R. J. Donnelly, C. E. Swanson, J. Fluid. Mech. 173, 387 (1986).

[17] S.K. Nemirovskii, V. V. Lebedev, Sov. Phys. JETP 57, 1009 (1983).

[18] K. W. Schwarz, Phys. Rev. B 31, 5782 (1985).

[19] D. Kivotides, Phys. Rev. B 78, 224501 (2008).

[20] D. Kivotides, Phys. Rev. B 77, 174508 (2008).

[21] D. Kivotides, J. Fluid Mech. 668, 58 (2011).

[22] A. Leonard, Phys. Fluids 6, 765 (1994).

[23] D. Kivotides, Phys. Rev. Fluids 3, 033701 (2018).

[24] J.-Z. Wu, H.-Y. Ma, M.-D. Zhou Vorticity and Vortex Dynamics, Springer (2009).

[25] M.V. Melander, F. Hussain, Fluid Dyn. Res. 13, 1 (1994). 
[26] D. Kivotides, C.F. Barenghi, D.C. Samuels, Science 290, 777 (2000).

[27] S. Leibovich, Ann. Rev. Fluid Mech. 10, 221 (1978).

[28] S.K. Nemirovskii, J. Low Temp. Phys. 171, 504 (2013).

[29] T. Zhang, S.W. Van Sciver, J. Low Temp. Phys. 138, 865 (2005).

[30] T.V. Chagovets, S.W. Van Sciver, Phys. Fluids 23, 107102 (2011).

[31] S.R. Stalp, L. Skrbek, R.J. Donnelly, Phys. Rev. Lett. 82, 4831 (1999). 